\section{A less fit flu virus}

\section{By Tim Fulmer, Senior Writer}

A team of researchers from the State University of New York at Stony Brook has used a computer-guided approach to engineer live attenuated influenza strains that protect mice from wild-type flu virus. ${ }^{1}$ The researchers think the strategy could lead to quicker, more efficient ways of producing live attenuated influenza vaccines than the method used to generate strains for the one marketed product, the seasonal FluMist vaccine.

Traditional techniques for generating live attenuated viral strains rely on identifying spontaneous mutations occurring in the viral genome. This typically involves extensive passage of the virus through cell or tissue culture under suboptimal conditions. The process can require months or even years to complete, and success is far from guaranteed-the result could be a strain that is neither genetically stable nor sufficiently attenuated for use in a human vaccine.

The Stony Brook researchers set out to develop a more efficient approach to viral attenuation (see Figure 1, “Computationally attenuating the flu virus"). They developed a computer-aided rational design strategy that rapidly generates influenza strains without the need for extensive passage through cell lines. They dubbed their method synthetic attenuated virus engineering (SAVE).

SAVE relies on a phenomenon called codon-pair bias to generate an attenuated strain that exhibits less viral protein expression than the wild-type strain but retains the wild-type amino acid sequences, thus retaining all viral epitopes that are recognized by the host immune system.

SAVE generates a recombinant form of the wild-type virus by rearranging the codons that make up the genome so that evolutionarily deoptimized codons are adjacent. The resulting shuffled genome still encodes wild-type proteins, but the deoptimization process makes translation of those proteins much less efficient than in a wild-type strain. The result is a virus that produces lower levels of the proteins necessary for viral replication, which directly attenuates the strain.

In previous work, the group showed that SAVE could produce live attenuated poliovirus strains that elicited sufficient antibodies to protect mice from death or paralysis following a lethal poliovirus challenge. ${ }^{2}$

The open question was whether SAVE could be extended to other viral types. To answer it, a team led by Eckard Wimmer and Steffen Mueller, professors in Stony Brook's Department of Molecular Genetics and Microbiology, decided to use SAVE to attenuate the influenza virus.

With the help of a computer program, the researchers first generated a panel of mutant influenza strains with deoptimized codon pairs in one, two or three genes that encode proteins important for viral replication and assembly-nucleoprotein $(N P)$, polymerase subunit B1 (PB1) and hemagglutinin (HA).

All of the mutant strains formed plaques and grew in vitro, though with about 10 -fold lower titers than those for wild-type influenza virus.

The next question was whether the strains would be sufficiently attenuated in vivo. The researchers focused on one of their mutant strains$\mathrm{PR} 8^{3 \mathrm{~F}}$ - which carries deoptimized codon pairs in all three genes.

Mice infected with PR8 ${ }^{3 \mathrm{~F}}$ showed no observable symptoms or weight loss except for a small delay in weight gain compared with mock-infected control animals. In contrast, mice infected with wildtype influenza showed a higher viral load, developed severe symptoms with rapid weight loss and did not survive more than five days.

Having shown that the $\mathrm{PR} 8^{3 \mathrm{~F}}$ strain was indeed attenuated in vivo, the researchers finally looked at whether it could protect against challenge by wild-type influenza.

In $80 \%$ of mice that received a single intranasal vaccination of $\mathrm{PR} 8^{3 \mathrm{~F}}$ prior to challenge with wild-type influenza, there was no detectable virus in the lungs. Comparatively, mock-vaccinated animals subjected to the same challenge had viral titers of about $10^{7}$.

The findings were published in Nature Biotechnology.

\section{Optimizing deoptimization}

The Stony Brook researchers now are focused on optimizing the attenuation strategy. "With proof of principle in hand, one of our current goals is to more clearly define how the location and number of deoptimized codon pairs in the influenza genome regularly correlates with the degree of strain attenuation," corresponding author Mueller told SciBX.

"That information should help us generate additional attenuated strains for potential vaccine use and also be useful for presenting to regulatory agencies that want evidence that our strategy can produce stable strain attenuation," he added.

Proof of strain stability will be essential moving forward, according to George Kemble, VP of R\&D at the MedImmune LLC subsidiary of AstraZeneca plc. "Much work is still needed to show that these attenuated strains are genetically stable and avoid compensating changes anywhere during the vaccine manufacturing process that could lead to an increase in virulence," he said.

MedImmune markets FluMist for children and adults ages 2-49.

Mueller said the odds of increases in virulence are vanishingly small. He told SciBX the number of deoptimized codon pairs introduced into a strain can be quite large, "which could make genetic reversion of our attenuated strains to more virulent strains very unlikely, perhaps nearly impossible."

In the PR8 ${ }^{3 \mathrm{~F}}$ strain, the deoptimization process led to the introduction of 314,236 and 353 mutations into the $N P, P B 1$ and $H A$ genes, respectively. By comparison, a minimum of 10 mutations in 4 genes are sufficient to generate the fully attenuated phenotype of one of MedImmune's master strains used to create FluMist. ${ }^{3-5}$

In the Nature Biotechnology paper, the authors wrote that vaccines based on their attenuation strategy "could be generated within weeks 


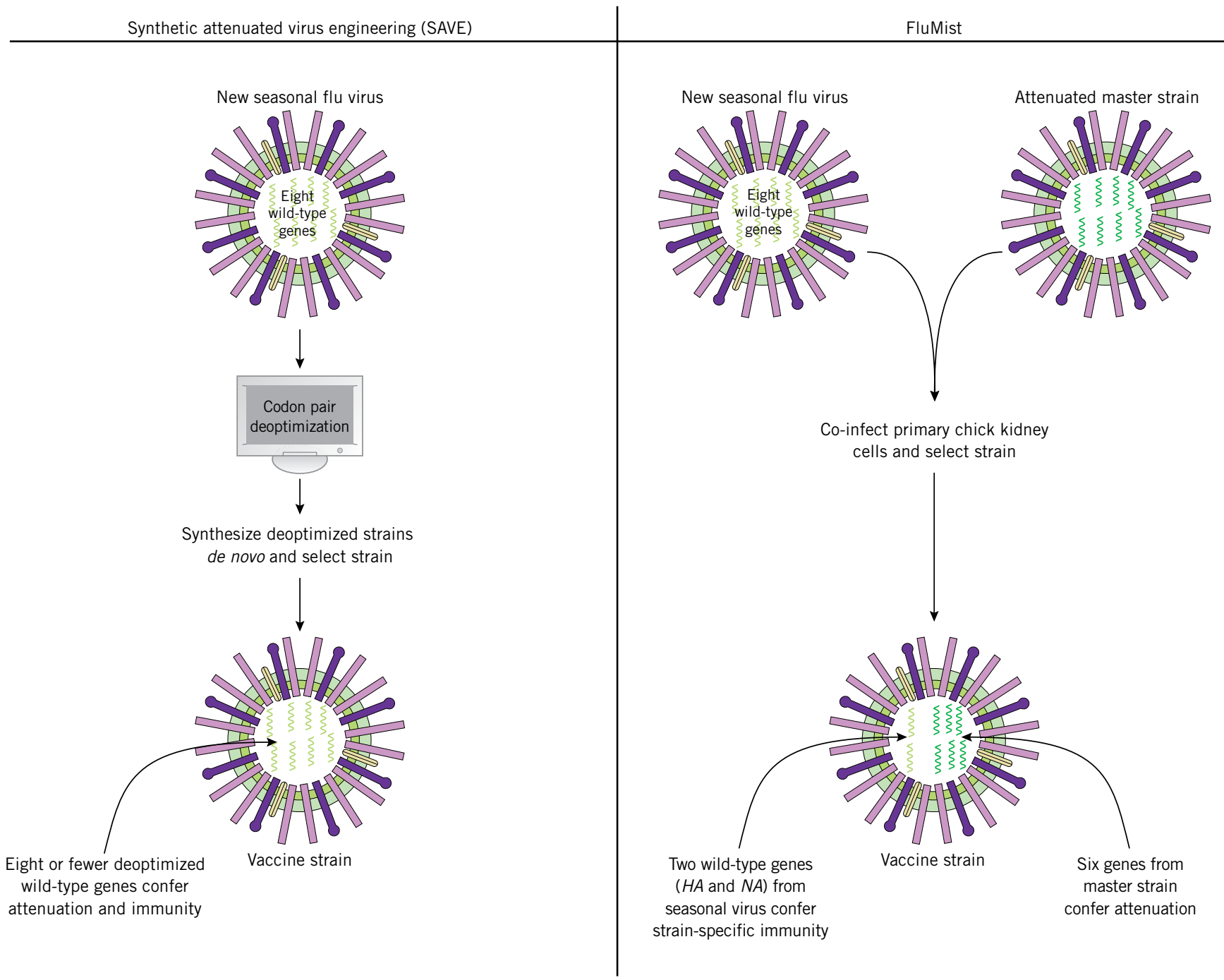

Figure 1. Computationally attenuating the flu virus. Synthetic attenuated virus engineering (SAVE) is intended to be a more efficient method of designing live attenuated influenza vaccines compared with the technique used to generate FluMist. Both SAVE and FluMist begin with the identification and sequencing of a recently emerged influenza virus. Then the two approaches diverge.

(I) SAVE applies the computational principle of codon-pair deoptimization to the wild-type sequence of the new flu virus, generating alternate viral genomes that consist of reshuffled codons. When done properly, the reshuffling procedure produces new viral genomes that express the same eight wild-type proteins at lower levels than the wild-type strain, resulting in strain attenuation. The alternate genomes are then synthesized de novo and tested for attenuation properties in vitro and in vivo. Based on that testing, a vaccine strain is chosen.

(II) FluMist begins by crossing the newly emerged strain with a previously attenuated master strain. The resulting vaccine strain consists of six genes from the attenuated master strain and two wild-type genes, hemagglutinin $(H A)$ and neuraminidase $(N A)$, from the new virus.

for any emerging influenza virus once its genome is known, although of course a further period of testing would be required before the vaccine could be used."

But according to Kemble, it is not yet clear if the codon-pair deoptimization strategy would be faster than the current method used to produce attenuated strains for the seasonal FluMist vaccine.

"It's important to realize that we don't begin every year with the attenuation of a brand-new wild-type influenza strain, which could indeed be time consuming using traditional attenuation techniques. Instead, we have highly stable attenuated master strains into which we insert variants of the two genes that are targeted by the seasonal flu vaccine. That requires significantly less time than if we started with the attenuation of the wild-type flu strain each year," he said.

Mueller's team now plans to study the attenuated strains in ferrets, which are better models of human influenza infection than mice.

The paper's findings are covered by patents and are available for licensing from Stony Brook.

Fulmer, T. SciBX 3(27); doi:10.1038/scibx.2010.817

Published online July 15, 2010 


\section{REFERENCES}

1. Mueller, S. et al. Nat. Biotechnol.; published online June 13, 2010; doi: $10.1038 / \mathrm{nbt} .1636$

Contact: Steffen Mueller, State University of New York at Stony Brook, Stony Brook, N.Y.

e-mail: smueller@ms.cc.sunysb.edu

2. Coleman, J. et al. Science 320, 1784-1781 (2008)

3. Chen, Z. et al. Virology 345, 416-423 (2006)
4. Hoffmann, E. et al. J. Virol. 79, 11014-11021 (2005)

5. Ambrose, C. et al. Influenza Other Respi. Viruses 2, 193-202 (2008)

COMPANIES AND INSTITUTIONS MENTIONED

AstraZeneca plc (LSE:AZN; NYSE:AZN), London, U.K.

Medlmmune LLC, Gaithersburg, Md.

State University of New York at Stony Brook, Stony Brook, N.Y. 